\title{
THE CONCEPT OF BEAM LINES FROM RHODOTRON FOR RADIATION TECHNOLOGIES
}

\author{
S. Korenev, STERIS Corporation, Libertyville, IL 60048, USA
}

\section{Abstract}

Among of main requirements to electron beam for industrial radiation technologies the variation of absorbed doses in irradiated product and kinetic energy of electrons is very important for increasing of efficiency of processes. The CW electron accelerator Rhodotron has technical opportunities for design of multi-lines system for radiation lines in industrial scale. The variant of multilines system on the basis of Rhodotron is considered in the report. The variation of current beam and time characteristics with multistage kinetic energy allows realizing this concept.

\section{INTRODUCTION}

The radiation technologies have different requirements on the dependence of radiation process [1]. The variation of absorbed doses can be realize using many different methods including the speed of conveyer (time of irradiation), beam current, kinetic energy. For using of multi-line's system with few beams, the variant of lines with different kinetic energy was considered for Rhodotron. Rhodotron is described in paper [2]. The main problem for same throughput of irradiated product is variation of absorbed doses for same thickness of product.

The concept of beam lines from Rhodotron is considered in this paper.

\section{MAIN CONCEPT}

The main concept of new beam lines consists in the variation of pulse duration of beam current for lines with fix kinetic energy:

1. the using of one Rhodotron accelerator with system of distributions of beam- lines (see Fig.1);

2. the using of time diagram for distribution of electron beams ( see Fig.2).

3. the suing of effects of Rhodotron for receiving of beams with step distribution of kinetic energy for electrons ( see Fig.3,4).

The main goal of this concept consists .in the receiving of simple system for irradiation of product with different thickness and different absorbed dose. It is very important question for radiation technologies. The question of multi-lines system discussed on the basis of separated electron accelerators with different kinetic energy. The multi-line system on the basis of accelerators is complex system and difficult engineering task.

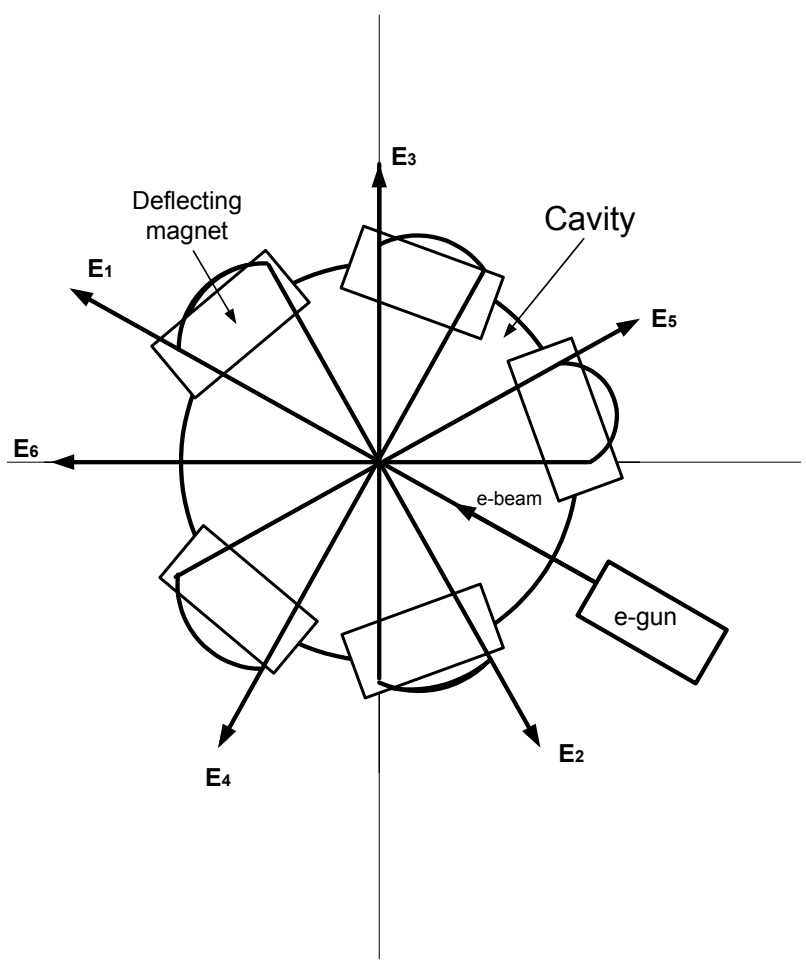

Figure 1: The main principle of multi-beams lines.

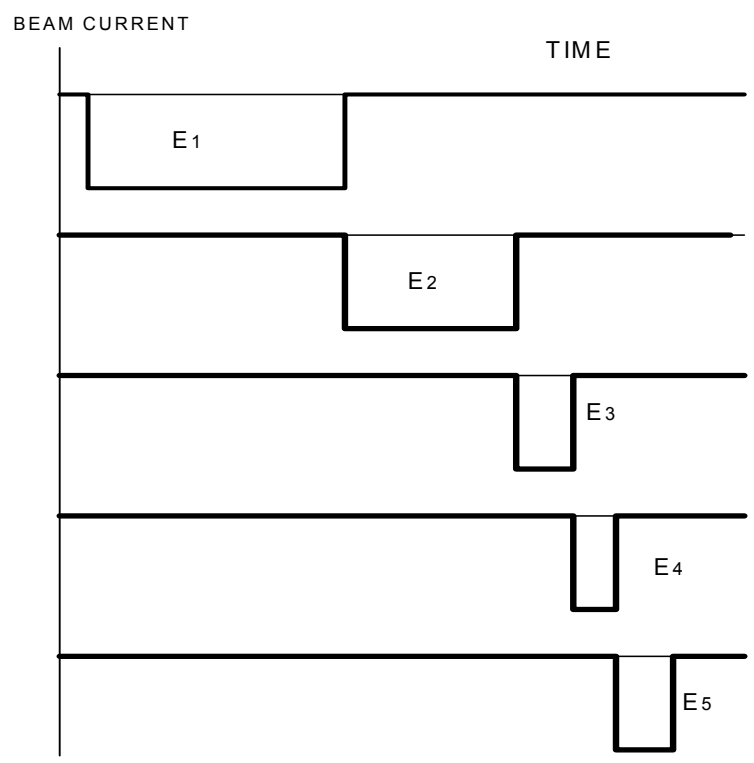

Figure 2: Time diagram of multi-line mode for Rhodotron 


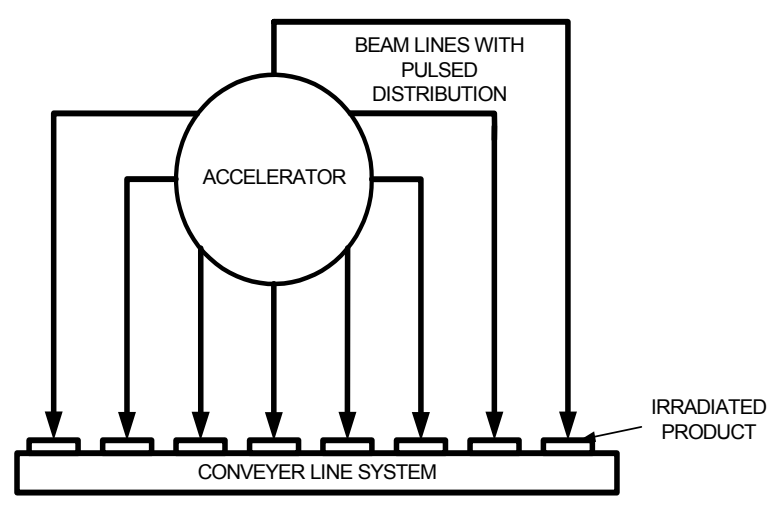

Figure 3: Multi-lines system.

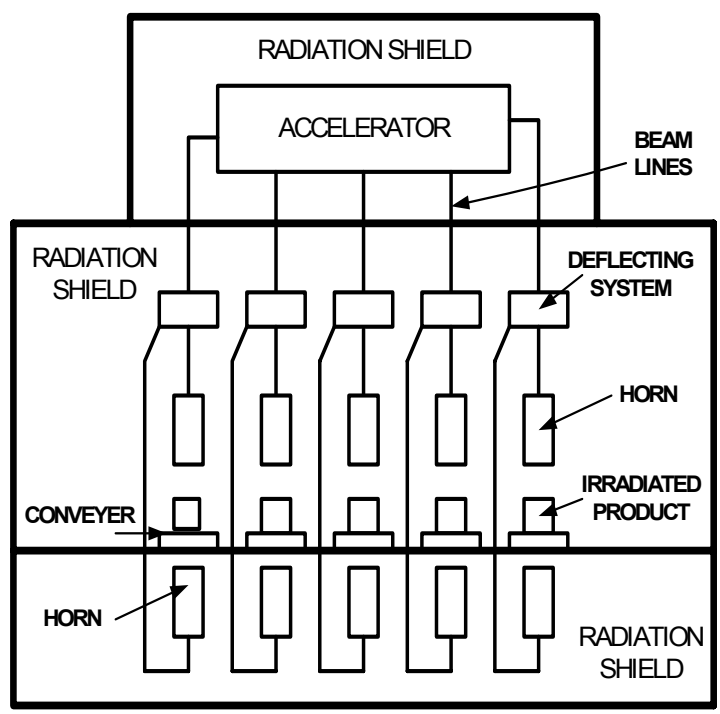

Figure 4: Structure of multi-lines with 2 sides of irradiation.

The absorbed doses $\mathrm{D}$ in irradiated product can be calculated using standard formula:

$$
D=\frac{E}{m}=\frac{E_{k} \cdot I \cdot t}{\rho \cdot S \cdot d_{o p t}},
$$

where: $E$ is absorbed energy, $m$ is mass of irradiated product, $E_{k}$ is kinetic energy (accelerating voltage), I is beam current, $\rho \mathrm{r}$ is density of irradiated product, $\mathrm{s}$ is square of irradiated product and $d_{\text {opt }}$ is optimal thickness of irradiated product.

The variation of kinetic energy allows the determination of optimal thickness for irradiated product. This type of accelerator allows to create the beam lines with step distribution of kinetic energy 1-2 MeV. The different thickness of irradiated product leads to increasing of efficiency of radiation process.
The variation of time of irradiation by regulation of pulse beam from Rhodotron allows to regulation of absorbed doses. The value of beam current for all lines is same.

The time diagram of supply of magnet system is same with pointed on Fig.2.

The using 2 sides of irradiation allows to increase the thickness of irradiated product. It is very critical for high density of materials.

The low level of absorbed doses about few kGy for many radiation technologies lead for simple variation of time beam in the beam -line. It is simple in the operation in comparison with speed of $\mathrm{v}$ conveyer. The other opportunity of variation of time irradiation is regulation by speed of conveyer line, but it is more technically difficult in comparison with regulation of time diagram for supply of magnet system for accelerator.

The consideration of magnet system shows the realization of suggested concept.

The simulation of magnetic field in the magnets of Rhodotron also shows the realistic approach. The development of current power supply with pulsed modes made big progress at last time with using of fast switches and it can be used for design of prototype of this system.

The question of beam dynamics at the process of study and primary consideration of non-stability of electron beam in cavity allows to hope on the small factor of nonstability of electron beam. The question of influence of space charge in the complex dynamics of beam in cavity is open. The effects of influence of secondary electrons in cavity need to consider more detail.

\section{CONCLUSION}

In conclusion we can point, that considered concept of beam multi-lines on the basis of electron accelerator Rhodotron is attempt for increasing efficiency of accelerator and radiation processes. The considered concept can be modified on dependence of radiation process. The suggested concept of multi-lines system on the basis of Rhodotron permits to autoimmunization of full system

Also I want to say thank you to many scientists for discussion of this topic, especially to John Masefield, Andrey Mishin, Vadim Dudnikoiv, Nikolay Mokhov.

\section{REFERENCES}

[1] R. Wood, A. Pikaev, "Applied radiation Chemistry: Radiation Processing”, W-I Publication, New York, 1994.

[2] J. Pottie, Nuclear Instruments and Methods in Physics Research B40/41, (1989), 943 